\title{
Trinity Detection System for SEM and FIB/SEM.
}

\author{
Petr Wandrol
}

Thermo Fisher Scientific, Materials \& Structural Analysis, Brno, Czech Republic

* Corresponding author: petr.wandrol@thermofisher.com

Detection of signal electrons belongs to key determinants of the image quality of a scanning electron microscope (SEM). Historically, signal electrons were detected in the specimen chamber. Nowadays, majority of modern field emission SEMs use an in-lens detection where one or multiple detectors located inside or above the final lens are employed to collect secondary (SE) and backscattered electrons (BSE). Electric or magnetic fields are used to create a final lens closely above or at the surface of a sample which attracts SE and BSE towards detectors above the pole piece.

NICol electron column [1] combines electric and magnetic fields of its final lens to enable high flexibility in the signal electron detection. The electric field is formed by a biased A-tube and is used to collimate and accelerate secondary and backscattered electrons towards the in-lens detectors. Strength of the magnetic field can be adjusted by the user to control distribution of SE and BSE at the in-lens detectors. The NICol column is equipped with a Trinity detection system containing up to three detectors positioned symmetrically around the primary beam axis. First two detectors (T1 and T2) are located in the final lens. Third detector, the T3, is higher up in the column as shown in Figure 1.

Primary imaging mode of the NICol column uses solely the electric field to guide signal electrons towards the Trinity detectors. The SE strike the T2 detector after passing through apertures in the pole piece and the T1 detector. SE emitted with lowest energies and along the optical axis are focused into the hole in the T2 and are collected by the T3 detector. Thanks to their higher energy, BSE are less sensitive to the electric field and hence hit the T1 detector. The in-chamber ETD detector collects the BSE emitted perpendicularly to the optical axis. Described detection principle as illustrated in Figure 2 provides maximum information about the sample by a single scan, namely its composition by the $\mathrm{T} 1$; topography and morphology by the T2 and ETD; surface details and voltage contrast by the T3. The T1 detector is uniquely positioned just above the pole piece so it always collects high number of BSE to produce images with high signal to noise ratio even at very low energies and currents of the primary beam. Compositional contrast delivered by the BSE depends on their angular and energy distribution. BSE emitted along the optical axis or at energies close to the energy of the primary beam ensure the strongest compositional contrast [2]. Trinity system offers several ways of maximizing the compositional contrast. One of them is selecting a particular working distance at which the electric field focuses the SE into the hole in the T2 and BSE emitted along the optical axis are collected instead. This is illustrated by the T2 image in Figure 3 where main components of a lithium ion battery cathode, the active material, the carbon filler and the solid electrolyte interface layer, are clearly recognizable. Contrast change from compositional to topographical is illustrated by T1 and ETD images in Figure 3 where BSE emitted at larger angles from the optical axis are collected. Low energy SE image collected by the T3 adds information about surface details and voltage contrast of the solid electrolyte interface layer. All four images were collected simultaneously to minimize irradiation of a beam sensitive sample.

In conclusion, the Trinity detection system enables unique flexibility of the signal electron collection and hence maximizes amount of information about the sample provided to the end user. 


\section{References:}

[1] D Wall et al, Microscopy and Microanalysis 20 (2014), p. 1102

[2] P Wandrol et al., Microscopy and Microanalysis 22 (2016), p. 576

[3] Images courtesy of T. Kazda, Brno University of Technology.
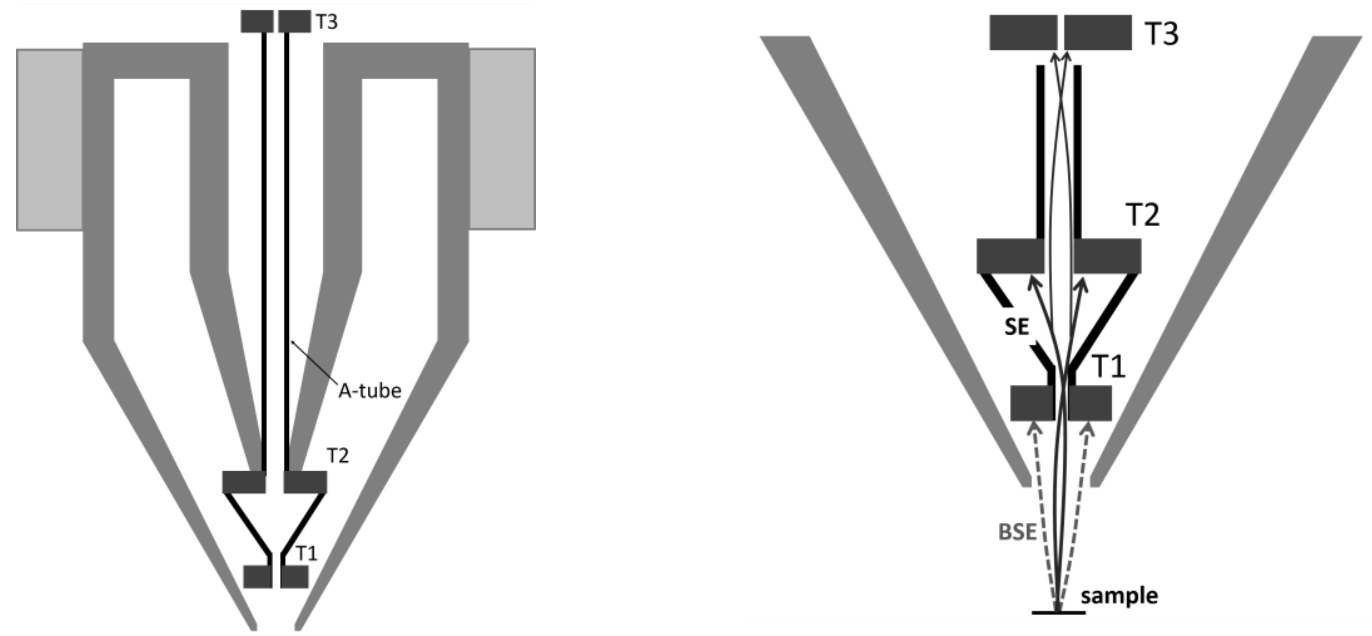

Figure 1. Schematic drawing of the NICol Figure 2. Illustration of the SE and BSE trajectories in the column with the Trinity detection system.
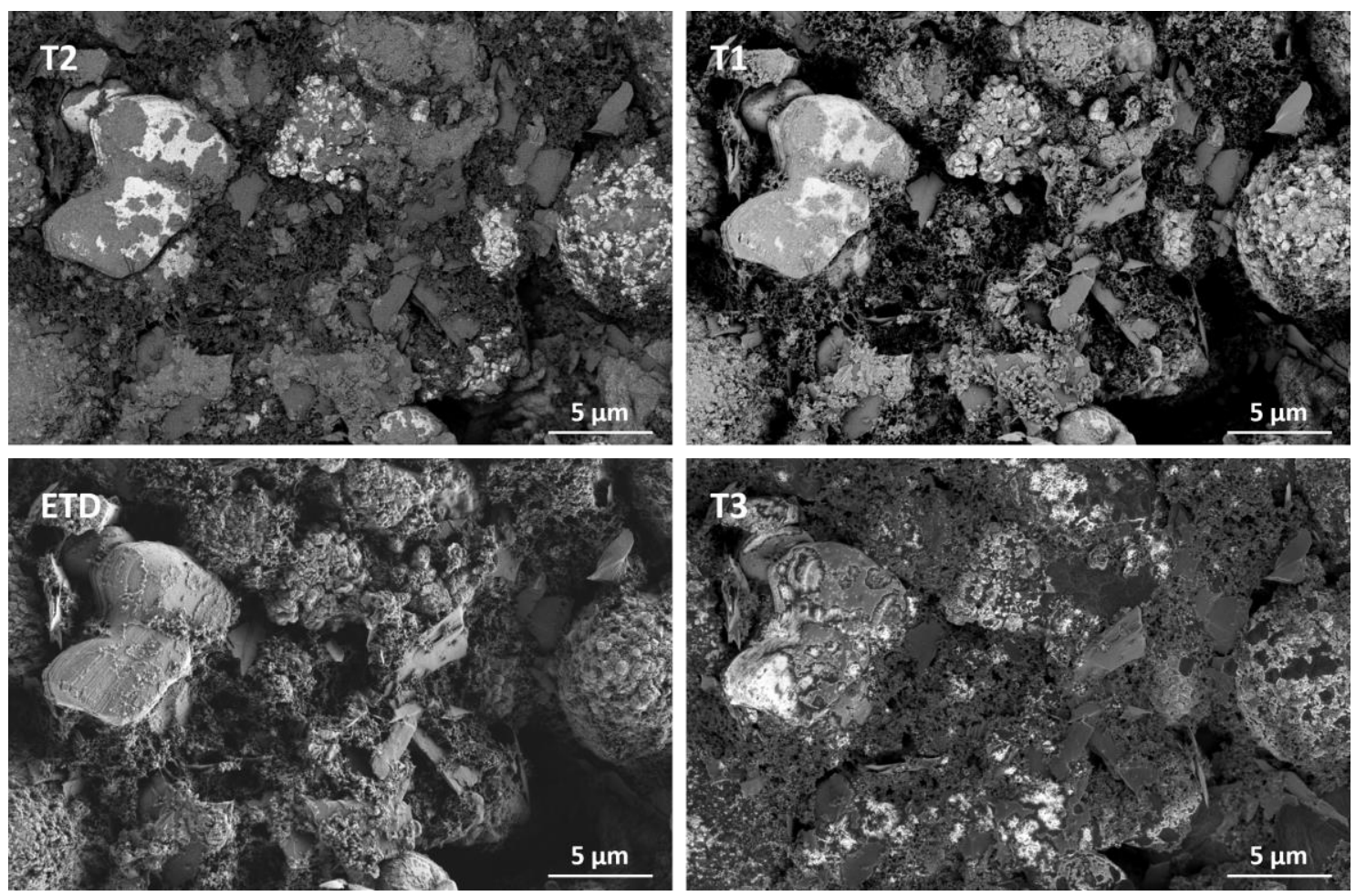

Figure 3. Lithium ion battery cathode imaged by BSE emitted from different angles from the Optical axis (T2 - parallel, ETD - perpendicular) and by low energy SE (T3). 